\title{
Erratum to: Constructal Design of Elliptical Conduits for Cooling of Gas Turbine Blades with External Thermal Barrier Coating
}

\author{
C. Bosc ${ }^{1 *}$, G. Lorenzini ${ }^{2}$, L. A. O. Rocha ${ }^{3 * *}$, F. R. Centeno ${ }^{3 * * *}$, and F. Gutierrez ${ }^{1 * * * *}$ \\ ${ }^{I}$ UIDET-IAME, National University of La Plata (UNLP), Department of Mechanical Engineering, \\ 1 and 47 Street, La Plata, CP 1900 Argentina \\ ${ }^{2}$ Dipartimento di Ingegneria e Architettura, Università degli Studi di Parma, \\ Parco Area delle Scienze 181/A, 43124 Parma, Italy \\ ${ }^{3}$ UFRGS-Federal University of Rio Grande do Sul, Department of Mechanical Engineering, Sarmento \\ Leite Street 425, Porto Alegre, 90050-170 Brazil
}

DOI: $10.1134 / \mathrm{S} 1810232820040153$

The list of authors: read not

C. Bosc, L. A. O. Rocha, F. R. Centeno, and F. Gutierrez, but

C. Bosc, G. Lorenzini, L. A. O. Rocha, F. R. Centeno, and F. Gutierrez

The original article can be found online at

https://doi.org/10.1134/S1810232819040064

\footnotetext{
${ }^{*}$ E-mail: cristian.bosc@ing.unlp.edu.ar

${ }^{* *}$ E-mail: luizrocha@mecanica.ufrgs.br

${ }^{* * *}$ E-mail: frcenteno@mecanica.ufrgs.br

***** E-mail: fernando.gutierrez@ing.unlp.edu.ar
} 